\title{
The use of hides to reduce acute stress in the newly hospitalised domestic cat (Felis sylvestris catus)
}

by Buckley, L.A. and Arrandale, L.

Copyright, Publisher and Additional Information: This is the author accepted manuscript. The final published version (version of record) is available online via Taylor \& Francis Please refer to any applicable terms of use of the publisher.

DOI: http://dx.doi.org/10.1080/17415349.2017.1301632

Harper Adams

University 
The use of hides to reduce acute stress in the newly hospitalised domestic cat (Felis sylvestris catus)

Buckley $^{1} \pm$, L. A. and Arrandale ${ }^{1}$, L.

${ }^{1}$ Veterinary Nursing Unit, Department of Animal Production, Welfare and Veterinary Sciences, Harper Adams University, Newport, Shropshire. TF1o 8NB

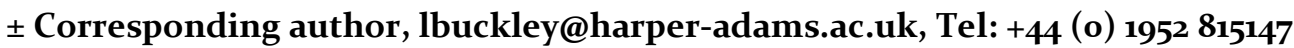

1. Abstract

The aims of this study were to identify if newly hospitalised cats would both use a hide, and show a reduction in stress levels when one was provided. Healthy cats attending a clinic for elective neutering were randomly allocated to either the Hide group $(n=15)$ or the No Hide group $(n=15)$. Temperature, heart and respiration rates were recorded on admission and after 20 minutes hospitalisation. Cats were cat stress scored (q 2 minutes) and their location with the kennel scored (q 30 seconds) over this 20 minute period.

Cats provided with a hide spent more time in the kennel location containing the hide $(\mathrm{P}<0.001)$, and used this enrichment primarily to hide inside $(\mathrm{P}<0.001)$. Compared to the baseline measurement, only Hide cats showed a small reduction in heart $(\mathrm{P}<0.001)$ and respiration $(\mathrm{P}<0.001)$ rates after 20 minutes. Cat stress score decreased in both groups of cats, but was significantly lower in Hide cats than No Hide cats after 20 minutes $(\mathrm{P}=0.002)$.

It is concluded that Hides are utilised and result in rapidly identifiable but small reductions in indices of stress in the newly hospitalised cat; thus, should be considered for use with this inpatient demographic.

2. Introduction

There are around 10,322,995 owned cats in the UK (Murray, et al., 2010) and it is expected that many will be hospitalised at a veterinary practice at least once during their lives (e.g. for neutering). Cats show physiological (Quimby et al., 2011; Nibblett et al., 2015) signs of stress when examined at the veterinary clinic, and will try to hide during examination (Nibblett et al. 2015). Trevorrow (2013) suggests the use of a hide to reduce the stress of hospitalised cats, with anxious or fearful cats particularly benefitting from this type of enrichment (Ellis, 2009). However, there is a lack of research that examines the use of hides on indices of either acute or chronic stress in the hospitalised feline patient.

Previous research to reduce captive housed feline stress levels has been focused within rescue and boarding catteries, and in laboratory settings (for example, see: Kry and Casey, 2007; Smith et al., 1994; and Carlstead et al.,1993). The use of a hiding enrichment has been demonstrated to reduce behavioural and physiological signs of stress in cattery - housed cats (e.g. Gourkow and Fraser, 20o6; Vinke et al., 2014, but see: Moore and Bain, 2013). Cats that can hide have reduced cortisol levels (Carlstead et al., 1993) and will utilise other items (e.g. litter trays) as hide substitutes where hides are not formally provided (Vinke et al., 2014). This suggests that hiding is an adaptive behaviour that cats are motivated to perform in stressful situations. However, cattery based studies demonstrate a reduction in stress over several days (e.g. 3 days, Moore and Bain, 2013; Vinke et al., 2014) but most hospitalised cats are housed for short periods of less than one day (e.g. for elective neutering). Thus, for hides to be beneficial to these cats, it is necessary to identify how quickly (if at all) hides will result in a measurable decline in acute stress levels of the feline inpatient. 
The aims of this study were to identify:

1. Whether (and how) cats would utilise a hide when provided with one.

2. If provision of a hide rapidly reduced the stress levels of newly hospitalised cats.

It was predicted that hides would be used by the cats and that their provision would result in a reduction in the levels of various behavioural and physiological indices of stress.

3. Materials and methods

3.1. Subjects, facility and treatment groups

The study took part at a mixed practice private veterinary practice (Tern Vets, Market Drayton, Shropshire, UK). The practice had separate wards for cats and dogs and the cat ward was used for this study. The cat kennels were arranged in one row and none of the kennels faced onto another kennel.

Thirty clinically healthy cats (aged between $5-7$ months, males: $\mathrm{n}=13$; females $=12$ ) admitted for routine elective neutering surgery were used. Cats were allocated to one of the two treatment groups based on the order of admittance (A, B, A, B, A, B, etc) to the clinic. The two treatment groups were:
A. $\quad$ No Hide cats $(\mathrm{n}=15)$.
B. Hide cats $(\mathrm{n}=15)$.

The No Hide cats (the control group) were provided only with a plastic, metal - bar fronted kennel $(85 \mathrm{~cm}$ (long) $\times 60 \mathrm{~cm}$ (wide) $\times 70 \mathrm{~cm}$ (height)) that was lined with newspaper. The experimental group, were provided with the same environment as the No Hide cats, plus a cardboard box (40cm (long) $\mathrm{x}$ $25 \mathrm{~cm}$ (wide) $x 25 \mathrm{~cm}$ (height)) in one corner of the kennel. The corner the box was placed in the same between cats. The box was positioned with the entrance facing into the middle of the kennel to allow the cat's behaviour to be observed at all times.

\subsection{Experimental protocol}

To answer the first aim (use of hide), the kennel was visually divided into four equal sized quadrants (A, $\mathrm{B}, \mathrm{C}$ and $\mathrm{D}$ ) and the cardboard box (Hide cats only) was always placed in quadrant $\mathrm{A}$. To answer the second aim (stress level reduction), temperature, heart and respiration rates were used as the physiological measurements of stress. The Cat Stress Score (see: Kessler and Turner, 1997) was used to measure behavioural signs of stress in the cats.

Each cat had its temperature, heart and respiration rate (collectively TPR) taken on admittance to the veterinary clinic. The cat was then placed into the kennel and directly observed by the experimenter. The cat's location with the kennel (which quadrant it was in) and interaction with the hide (on top versus inside) was recorded every 30 seconds and the stress score every two minutes. After twenty minutes, the cat was removed from the kennel and the TPR repeated.

\subsection{Statistical analysis}

All data was analysed using the Genstat (VSN International, Hemel Hempstead, UK) statistical package (version 14). Cat stress score, and all physiology data was normally distributed. Therefore, this data is reported using means and standard error of the mean (SEM) and analysed using the Student's T - test (inter - group data) and the Matched Pairs T - test (intra - group data). The quadrant and hide usage data has been expressed at time spent (number of data points observed doing $\mathrm{X}$ over the 20 minute period). The quadrant location and the hide use data was excessively positively skewed $(>1)$ and was 
therefore analysed using the Kruskal Wallis and Wilcoxon Matched Pairs tests and reported using medians and the inter - quartile range.

\subsection{Ethical considerations}

The study was approved as per the Harper Adams Ethic committee protocol for undergraduate research projects. The primary experimental modification applied (the hide) was expected to have only positive welfare effects for the cats concerned. The data collection techniques were non - invasive or relatively low invasive (e.g. temperature taking).

4. Results

4.1. Do cats use a hide when a hide is provided?

Cats provided with a hide were significantly more likely to spend time in the hide - containing quadrant A than the other 3 quadrants $\left(\mathrm{H}_{15}=25.87, \mathrm{P}<0.001\right)$, whereas cats not provided with a hide did not show a preference for any of the quadrants $\left(\mathrm{H}_{15}=0.61, \mathrm{P}=0.893\right)$. See figure 1 .

\section{**FIGURE ONE GOES HERE**}

4.2. How do cats interact with the hide when a hide is provided?

When interacting with the hide, the Hide cats were significantly $\left(\mathrm{U}_{15}, 15=7.5, \mathrm{P}<0.001\right)$ more likely to go inside the box than they were to sit on top of it. The median (I.Q.) time spent inside the box over the 20 minute observation period was $9(3.3-8.5)$ minutes, whereas the median (I.Q.) time spent on top of the box was o $(\mathrm{o}-\mathrm{o})$ minutes. Only two cats were observed to utilise the top of the box (one for only 0.5 minutes, the other for 7 minutes).

\subsection{Effect of hide provision on the behavioural signs of stress}

Cats in both treatment groups showed a significant decline in their cat stress score from the baseline measurement (o minutes) at both 10 minutes (No Hide cats: $\mathrm{T}_{14}=2.33, \mathrm{P}=0.033$; Hide cats: $\mathrm{T}_{14}=3.85 . \mathrm{P}$ $=0.002$ ) and 20 minutes (No Hide cats: $\mathrm{T}_{14}=2.5, \mathrm{P}=0.025$; Hide cats: $\mathrm{T}_{14}=11.03, \mathrm{P}<0.001$ ) post - entry to the cat kennel.

The Cat Stress Scores did not differ significantly between the Hide cats and the No Hide cats at the start of the observation period $\left(\mathrm{T}_{28}=0.64, \mathrm{P}=0.53\right)$ or at the 10 minute point $\left(\mathrm{T}_{28}=-0.45, \mathrm{P}==0.653\right)$. However, by the end of the observation period (20 minutes) Hide cats showed a significantly lower stress score $\left(\mathrm{T}_{28}=-3.38, \mathrm{P}=0.002\right)$ than No Hide cats. See figure 2 .

\section{**FIGURE TWO GOES HERE**}

\subsection{The effect of hide provision on heart rate}

The heart rates of the cats differed between groups at both $\mathrm{o}$ and 20 minutes. The heart rate of the Hide cats was significantly higher $\left(T_{28}=2.28, P=0.030\right)$ than the No Hide cats: Hide cats: $151( \pm 2)$ heart beats per minute (BPM); No Hide cats: $143( \pm 3)$ BPM at o minutes. However, after 20 minutes, the Hide cats' heart rate was now significantly lower $\left(\mathrm{T}_{28}=-2.48, \mathrm{P}=0.019\right)$ than the No Hide cats' heart rate: Hide cats: 131 $( \pm 3)$ BPM; No Hide cats: $142( \pm 3)$ BPM.

Intra-group, only the Hide cats showed a significant reduction in heart rate over the 20 minute period, whereas, the No Hide cats had a similar heart rate after this period (Hide cats: $\mathrm{T}_{14}=6.98 ; \mathrm{P}<0.001$; No Hide cats: $\mathrm{T}_{14}=0.65, \mathrm{P}=0.528$ ).

4.5. The effect of hide provision on respiration rate 
The respiration rate of the cats in the Hide and No Hide groups was similar at o minutes $\left(\mathrm{T}_{28}=0.66, \mathrm{P}\right.$ 0.514): Hide cats: $46( \pm)$ respirations per minute (RPM); No Hide cats: $43( \pm 3)$ RPM. Intra-group, only the Hide cats respiration rate had decreased significantly by 20 minutes (Hide cats: $\mathrm{T}_{14}=7.22, \mathrm{P}<0.001$; No Hide cats: $\left.\mathrm{T}_{14}=0.65, \mathrm{P}=0.525\right)$. However, this change in respiration rate was very small and this was reflected in no significant difference in respiration rate being observed at 20 minutes between the Hide and No Hide cats $\left(\mathrm{T}_{28}=1.47, \mathrm{P}=0.153\right)$. The respiration rate at 20 minutes was: Hide cats: $36( \pm 2) \mathrm{RPM}$; No Hide cats: $41( \pm 2)$ RPM.

\subsection{The effect of hide provision on temperature}

The temperature of the cats in the Hide and No Hide groups was similar at both o minutes (Hide cats: $38.5 \pm 0.03^{\circ} \mathrm{C}$ : No Hide cats: $38.4 \pm 0.03^{\circ} \mathrm{C} ; \mathrm{T}_{28}=0.65, \mathrm{P}=0.518$ ) and 20 minutes (Hide cats: $38.4 \pm 0.03$ ${ }^{\circ} \mathrm{C}$; No Hide cats: $38.4 \pm 0.03^{\circ} \mathrm{C} ; \mathrm{T}_{28}=0.98, \mathrm{P}=0.334$ ).

\section{Discussion}

This study provides clear evidence that, in the short term, cats will hide in a hiding enrichment provided to cats immediately upon being hospitalised within the veterinary clinic. Furthermore, the study provides limited, but positive, evidence that hides will provide a rapid reduction in both physiological and behavioural indices of stress in the hospitalised cat. Thus it provides some support for the practical recommendations of both Ellis (2009), Trevorrow (2013), and the American Association of Feline Practitioners and the International Society for Feline Medicine (Ellis et al. 2013) that hides should be utilised to help facilitate feline patient wellbeing. Consideration should be given to also providing hides to cats immediately upon hospitalisation, rather than reserving them for use with cats being hospitalised for longer periods of time.

One clear limitation to this study is the very short time frame over which the researcher observed the hospitalised cats. This partially reflects clinical practice in some practices whereby patients are pre medicated and / or anaesthetised soon after being admitted to the clinic. However, it also results in clear experimental limitations. It should be noted that the reduction in all parameters was very small and it is not immediately clear whether such small changes have any biological relevance for the feline patient in terms of psychological wellbeing, reduced risk of procedure or anaesthetic - linked mortality or morbidity and further research would be beneficial in these areas. For example, the risk of an anaesthetic - related death in cats is much higher for cats than for humans ( $0.24 \%$ in cats but only 0.02 - $0.05 \%$ in humans (Brodbelt et al., 2007), but the contribution of psychological stressors (resulting in e.g. increased catecholamine release) to this risk is not known.

It would also be beneficial to study the effects of stress on hospitalised cats over a longer timeframe. For example, if the cats had been studied for 60 minutes, would a greater divergence in stress scores, heart and respiration rates been observed or would these parameters now be similar between cats? The work by Vinke et al. (2014) suggests that greater divergence would be observed as, in their study, it took 14 days for the cat stress score of No Hide cattery - housed cats to reach the same level as Hide cats. However, this remains to be demonstrated in the veterinary clinic. In the absence of this information though, it is safer to assume greater divergence and provide hides as a benign potential welfare enhancer, unless clinical need precludes the use.

In conclusion, hides are utilised by recently hospitalised cats and are associated with small, but positive effects on behavioural and physiological indices of stress and their use should be considered even in feline patients hospitalised for very short periods of time.

6. Acknowledgements 
This study was carried out part of Laura Arrandale's undergraduate studies and formed part of her final year honours dissertation. The authors would like to thank the staff at Tern Veterinary Group for allowing data collection to be undertaken at their Market Drayton clinic. The authors have no conflicts of interest to declare.

\section{References}

Carlstead, K., Brown, J. L. and Strawn, W. 1993. Behavioral and physiological correlates of stress in laboratory cats. Applied Animal Behaviour Science 38(2): 143 - 158

Ellis, S. 2009. Environmental enrichment: practical strategies for improving feline welfare. Journal of Feline Medicine and Surgery 11: 901 - 912

Ellis, S., Rodan, I., Carney, H. C., Heath, S., Rochlitz, I., Shearburn, L. D., Sundahl, E. and Westropp, J. L. 2013. AAFP and ISFM Feline Environmental Needs Guidelines. Journal of Feline Medicine and Surgery 15: $219-230$

Gourkow, N. and Fraser, D. 2006. The effect of housing and handling practices on the welfare, behaviour and selection of domestic cats (Felis sylvestris catus) by adopters in an animal shelter. Animal Welfare 15: $371-377$

Kessler, M. R. and Turner, D.C. 1997. Stress and adaptation of cats (Felis sylvestris catus) housed singly, in pairs and in groups in boarding catteries. Animal Welfare 6(3): $243-254$

Kry, K. and Casey, R. 2007. The effect of hiding enrichment on stress levels and behaviour of domestic cats (Felis sylvestris catus) in a shelter setting and the implications for adoption potential. Animal Welfare 16(3): $375-383$

Moore, A. M. and Bain, M. J. 2013. Evaluation of the addition of in - cage hiding structures and toys and timing of behavioural assessments with newly relinquished shelter cats. Journal of Veterinary Behaviour $8(6): 450-457$

Murray, J. K., Browne, W. J., Roberts, M. A., Whitemarsh, A. and Gruffydd-Jones, T. J. 2010. Number and ownership profiles of cats and dogs in the UK. Veterinary Record 166(6): 163-168

Nibblett, B. M., Ketzis, J. K. and Grigg, E. K. 2015. Comparison of stress exhibited by cats examined in a clinic versus a home setting. Applied Animal Behaviour Science 173: 68 - 75

Quimby, J. M., Smith, M. L. and Lunn, K. F. 2011. Evaluation of the effects of hospital visit stress on physiologic parameters in the cat. Journal of Feline Medicine and Surgery 13: 733 - 737

Smith, D. F., Durman, K. J., Roy, D. B. and Bradshaw, J. W. 1994. Behavioural aspects of welfare of rescued cats. The Journal of the Feline Advisory Bureau 31: 25 - 28

Trevorrow, N. 2013. Helping cats cope with stress in veterinary practice. Veterinary Nursing Journal 28: $327-329$

Vinke, C. M., Godin, L. M. and van der Leij, W. J. R. 2014. Will a hiding box provide stress reduction for shelter cats? Applied Animal Behaviour Science 16o: 86 - 93 
Figure 1: Median time spent in each quadrant $\mathrm{x}$ treatment group. The box was placed in quadrant ' $\mathrm{A}$ ' (Hide cats only). Nb. The error bars signify the inter-quartile range.

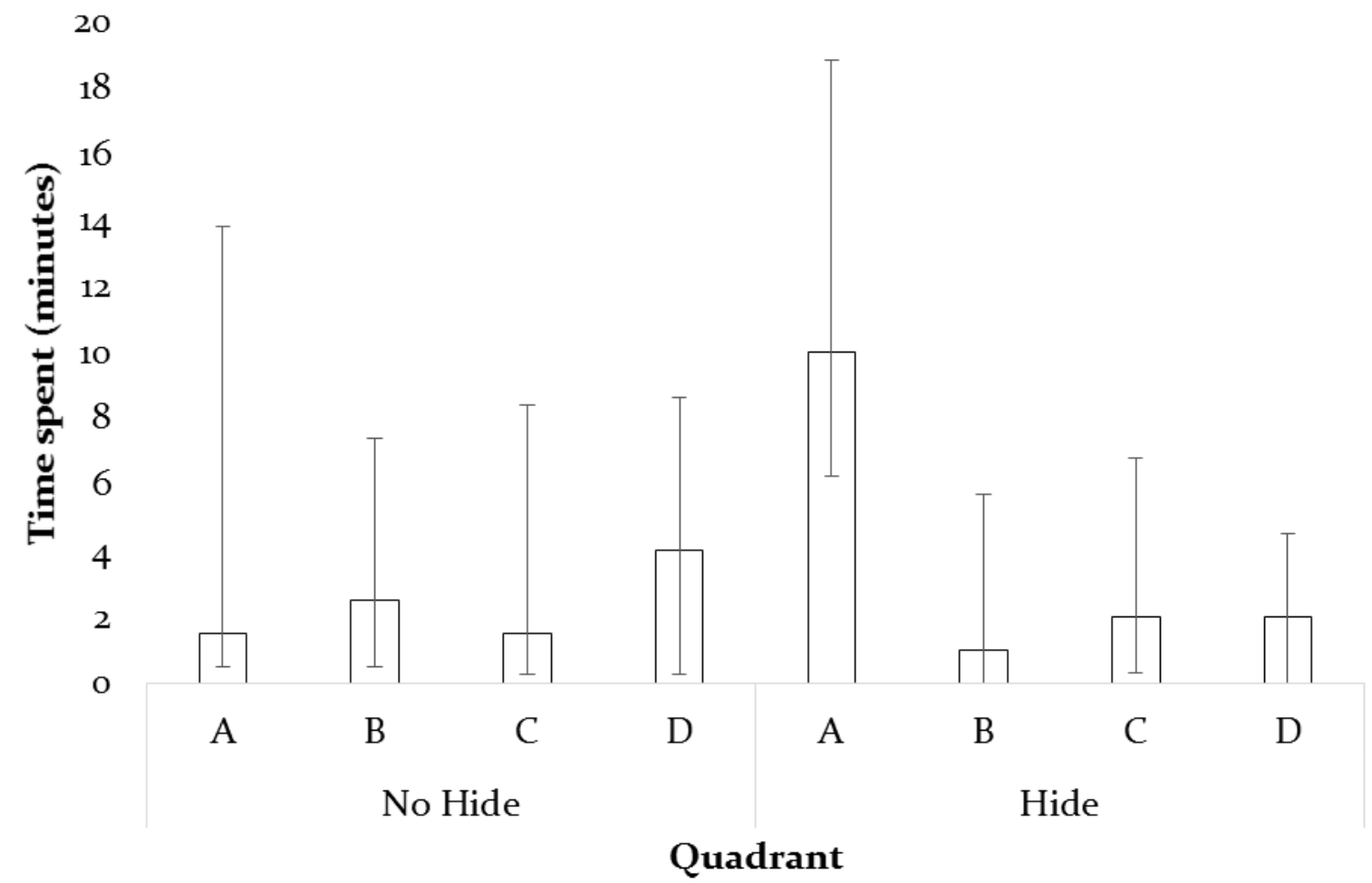

Figure 2: The effect of hide provision and time on the mean cat stress score. Nb. The error bars indicate the standard error of the mean.

$\multimap$ No Hide -- -- -Hide

6
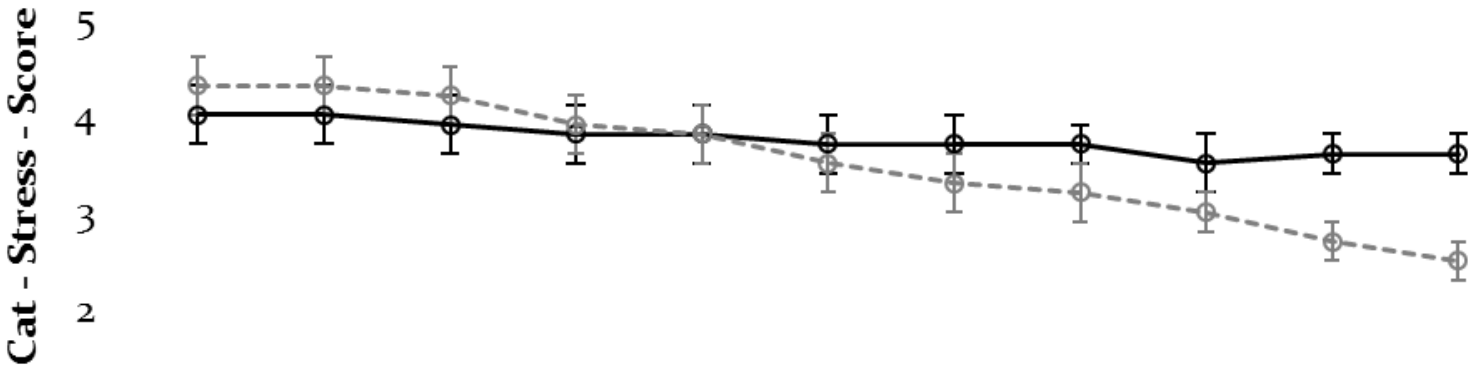

1

$\mathrm{O}$

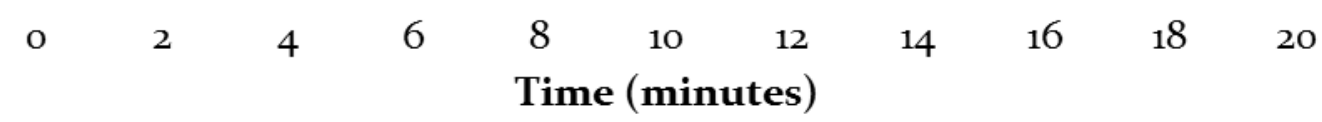

